\title{
Modelling of stratified gas-liquid two-phase flow in horizontal circular pipes
}

\author{
Paulo A.B. de Sampaio ${ }^{\text {a }}$, José L.H. Faccini ${ }^{\text {a,b }}$, Jian Su ${ }^{\text {b,* }}$ \\ ${ }^{a}$ Nuclear Engineering Institute, Brazilian Nuclear Energy Commission (CNEN), CP 68550, Rio de Janeiro 21945-970, Brazil \\ ${ }^{\mathrm{b}}$ Nuclear Engineering Program, COPPE, Universidade Federal do Rio de Janeiro, CP 68509, Rio de Janeiro 21945-970, Brazil
}

Received 2 February 2007; received in revised form 27 September 2007

Available online 4 December 2007

\begin{abstract}
This paper reports numerical and experimental investigation of stratified gas-liquid two-phase flow in horizontal circular pipes. The Reynolds averaged Navier-Stokes equations (RANS) with the $k-\omega$ turbulence model for a fully developed stratified gas-liquid twophase flow are solved by using the finite element method. A smooth interface surface is assumed without considering the effects of the interfacial waves. The continuity of the shear stress across the interface is enforced with the continuity of the velocity being automatically satisfied by the variational formulation. For each given interface position and longitudinal pressure gradient, an inner iteration loop runs to solve the non-linear equations. The Newton-Raphson scheme is used to solve the transcendental equations by an outer iteration to determine the interface position and pressure gradient for a given pair of volumetric flow rates. Favorable comparison of the numerical results with available experimental results indicates that the $k-\omega$ model can be applied for the numerical simulation of stratified gas-liquid two-phase flow.
\end{abstract}

(C) 2007 Elsevier Ltd. All rights reserved.

Keywords: Gas-liquid two-phase flow; Smooth stratified flow; $k-\omega$ model; Finite element method; Computational simulation

\section{Introduction}

Gas-liquid two-phase stratified flow in horizontal ducts is frequently encountered in practical applications such as nuclear reactors, oil and gas pipelines, steam generation and refrigeration equipment. The accurate prediction of pressure gradient and void fraction in gas-liquid two-phase stratified flow is of both scientific and technological interests. The mechanistic model due to Taitel and Dukler [1] has been widely used, which is a one-dimensional two-fluid model with closure relations for the wall and interfacial shear stresses calculated with single-phase flow correlations. However, the Taitel and Dukler model neglects the detailed velocity profile structure and calculates the wall

\footnotetext{
${ }^{*}$ Corresponding author. Tel.: +55 212562 8448; fax: +55 2125628444 .

E-mail addresses: sampaio@ien.gov.br (P.A.B. de Sampaio), faccini@ ien.gov.br (J.L.H. Faccini), sujian@con.ufrj.br (J. Su).
}

and interfacial shear stresses via empirical correlations based on the averaged velocities.

With the recent advent of high-speed computers and the development of advanced turbulence models, the computational fluid dynamics (CFD) techniques have been applied for the simulation of stratified gas-liquid two-phase flow. Shoham and Taitel [2] presented one of the early twodimensional numerical solutions of fully developed turbulent-turbulent gas-liquid flow in horizontal and inclined pipes. The gas phase was treated as bulk flow, while the liquid phase momentum equation in the bipolar co-ordinate system with an algebraic turbulent model was solved by using a finite difference method. Also using the bipolar co-ordinate system, Issa [3] modeled stratified flow, with a smooth interface surface, but solved the axial momentum equation in both gas and liquid phases with the standard $k-\varepsilon$ model. Wall functions were used in the solid boundaries. The results for flow in a $25.4 \mathrm{~mm}$ diameter pipe agree reasonably with predictions given by the mechanistic model 


\begin{tabular}{llll}
\hline Nomenclature & & \\
& & & \\
$A_{i}$ & coefficients & $\alpha_{2}$ & model parameter \\
$B_{i}$ & coefficients & $\beta$ & volumetric fraction \\
$C_{i}$ & coefficients & $\beta_{1}$ & model parameter \\
$c_{\mathrm{f}}$ & skin friction coefficient & $\beta_{2}$ & model parameter \\
$d$ & inner diameter of the pipe & $\Gamma$ & boundary \\
$\mathbf{e}$ & unit vector & $\varepsilon$ & dissipation rate \\
$F$ & mismatch function & $\epsilon_{\mathrm{f}}$ & liquid hold-up \\
$G$ & mismatch function & $\mu$ & fluid viscosity \\
$k$ & turbulent kinetic energy & $\mu_{t}$ & eddy viscosity \\
$\mathbf{n}$ & normal unit vector & $\rho$ & density \\
$N$ & finite element shape function & $\sigma_{1}$ & model parameter \\
$p$ & pressure & $\sigma_{2}$ & model parameter \\
$Q$ & volumetric flowrate & $\omega$ & specific dissipation rate \\
$S_{i}$ & source terms & $\Omega$ & domain \\
$u$ & velocity & & \\
$V$ & function space & $S u b s c r i p t s$ \\
$x$ & representing d $p /$ d $z$ & c & wall \\
$y$ & representing $y_{\text {int }}$ & f & liquid phase \\
$y_{\text {int }}$ & interface position & g & gas phase \\
$z$ & longitudinal co-ordinate & int & interface \\
$G r e e k$ & symbols & $\mathrm{s}$ & symmetry boundary \\
$\alpha_{1}$ & model parameter & & \\
\hline
\end{tabular}

of Taitel and Dukler [1]. Newton and Behnia [4] used a low Reynolds number $k-\varepsilon$ model that allows the solution of the turbulent-turbulent stratified flow problem. The numerical results shown are in good agreement with experimental data of a $50 \mathrm{~mm}$ diameter pipe [5] and indicate that the minor tuning of the wall damping functions performed has little effect on the results. More recently, stratified wavy two-phase flow has also been studied numerically [6-9]. Mouza et al. [10] employed a commercial CFD code to study horizontal wavy stratified two-phase flow in pipe and channel flows. However, the liquid film thickness was specified by available experimental data or correlation and thus not a part of the solution.

Two-phase gas-liquid flow in different geometries and flow regimes has been also investigated computationally. Akai et al. [11] applied a two-equation $k-\varepsilon$ model to twophase stratified fully developed turbulent-turbulent flow between parallel plates. The effects of interfacial waves on the flow field were formulated in terms of gas-liquid interfacial conditions. In a closely related work, Akai et al. [12] studied experimentally and analytically horizontal, co-current, stratified, air-mercury flow in a rectangular channel. Biberg and Halvorsen [13] studied analytically two-phase stratified laminar flow in a circular pipe and obtained interfacial and wall shear stress distributions. Banerjee and Isaac [14] performed a numerical study to determine the rate of evaporation of gasoline while flowing through an inclined two-dimensional channel. Two-phase vapor-liquid stratified flow was solved by using the volume-of-fluid
(VOF) method. Yap et al. [15] presented a numerical solution of an annular two-phase flow in a square channel using the level-set method. Numerical techniques to treat the sharp gas-liquid interface have been investigated intensively recently [16-23]. Turbulent interfacial gas-liquid flows have been also studied by using directed numerical simulation (DNS) [24] and large-eddy simulations (LES) [25].

In this work, we solve the Reynolds averaged NavierStokes equations (RANS) with the $k-\omega$ model for a fully developed stratified gas-liquid two-phase flow using the finite element method. The closure model was developed by Wilcox [26] and is considered substantially more accurate than $k-\varepsilon$ model in the near wall layers [27]. The main drawback of the model is that the $\omega$-equation shows a strong sensitivity to the values of $\omega$ in the freestream outside the boundary layer [28], which has largely prevented the $\omega$-equation from replacing the $\varepsilon$-equation as the standard scale-equation in turbulence modeling. However, it is expected that the $k-\omega$ model should have a better performance in the prediction of gas-liquid two-phase stratified flow, as no freestream boundary condition of $\omega$ is needed in the modeling. Following Issa [3] and Newton and Behnia [4], a smooth and horizontal interface surface is assumed without considering the interfacial waves. The continuity of the shear stress across the interface is enforced with the continuity of the velocity being automatically satisfied by the variational formulation. The mathematical model and the variational formulation are presented in the next 
section. The numerical techniques are then presented including the flow solver and the Newton-Raphson rootfinding scheme. The numerical solution is then verified for the single-phase flow over a wide range of Reynolds numbers. For each given interface position and longitudinal pressure gradient, an inner iteration loop runs to solve the non-linear equations. The Newton-Raphson scheme is used to solve the transcendental equations by an outer iteration to determine the interface position and pressure gradient for a given pair of volumetric flow rates. The numerical results are then compared with available experimental data.

\section{Two-phase stratified flow model}

Let us consider a fully developed stratified gas-liquid two-phase flow in a horizontal circular pipe. In view of the symmetry of the flow with respect to the vertical plane, only a half-pipe cross-section is considered in the present model. Fig. 1 shows schematically the open bounded domains occupied by the liquid and gas phases, which are denoted by $\Omega_{\mathrm{f}}$ and $\Omega_{\mathrm{g}}$, respectively. We consider that the volumetric flow rates of the phases, $Q_{\mathrm{f}}$ and $Q_{\mathrm{g}}$, are given.

The interface between the phases is assumed to be a horizontal plane. However, the interface position $y_{\text {int }}$ is unknown. In fact, it will be determined as a function of the given flow rates, pipe diameter and the physical properties of the liquid and gas phases. Referring to Fig. 1, the gas-liquid interface is represented by $\Gamma_{\text {int }}$, the symmetry boundary is denoted by $\Gamma_{\mathrm{s}}$ and the pipe wall is $\Gamma_{\mathrm{c}}$. We also

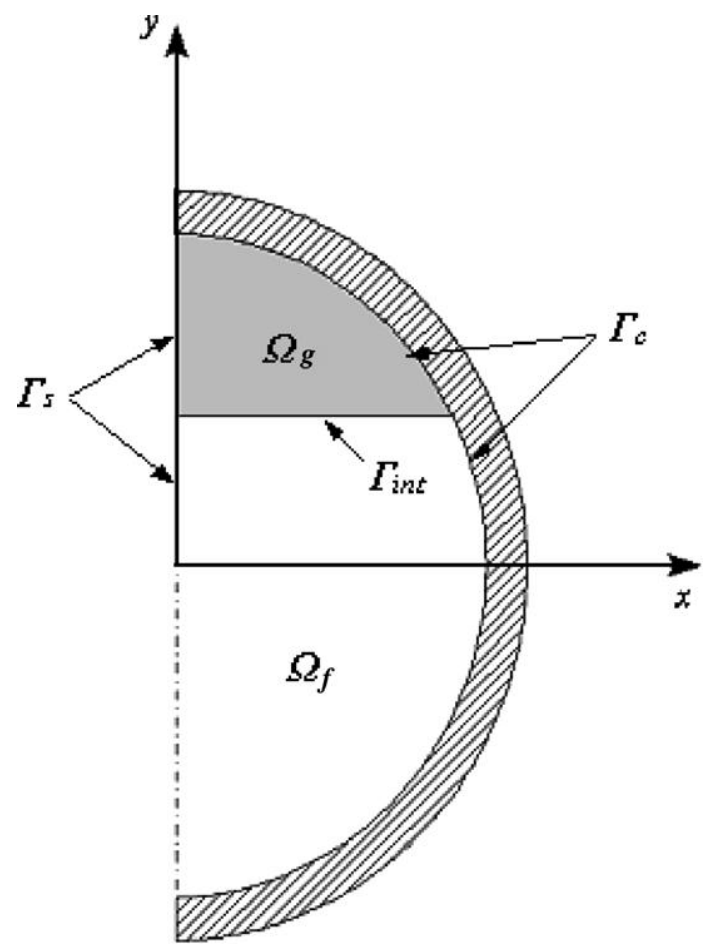

Fig. 1. Schematic representation of the pipe cross-section and computational domains. define the overall open bounded domain $\Omega=$ $\Omega_{\mathrm{f}} \cup \Omega_{\mathrm{g}} \cup \Gamma_{\text {int }}$.

The Reynolds averaged Navier-Stokes (RANS) approach is adopted to describe the turbulent flow in both phases. For developed turbulent flow, the two-phase flow model is described by the following equations, defined within each open bounded domain $\Omega_{i}(i=1$ meaning phase $f$ and $i=2$ phase $g$ ).

$$
\begin{aligned}
& \nabla \cdot\left(A_{i} \nabla u\right)-\frac{\mathrm{d} p}{\mathrm{~d} z}=0 \\
& \nabla \cdot\left(B_{i} \nabla k\right)-\beta_{2} \rho_{i} k \omega+S_{i}=0 \\
& \nabla \cdot\left(C_{i} \nabla \omega\right)-\beta_{1} \rho_{i} \omega^{2}+\frac{\alpha_{1} \omega}{k} S_{i}=0
\end{aligned}
$$

In the above equations the flow, with velocity $u$, is aligned to co-ordinate $z$. The kinetic energy of turbulence is represented by $k$ and the dissipation per unit turbulence kinetic energy is denoted by $\omega$. Because the flow is assumed to be fully developed, the same pressure gradient $\mathrm{d} p / \mathrm{d} z$ is considered for both phases. Note though, that like the interface position $y_{\text {int }}, \mathrm{d} p / \mathrm{d} z$ is an unknown variable that will be determined as a function of the given volumetric flow rates.

Other terms appearing in Eqs. (1)-(3) are $A_{i}=\mu_{i}+\mu_{t i}$, $B_{i}=\mu_{i}+\sigma_{2} \mu_{t i}, \quad C_{i}=\mu_{i}+\sigma_{1} \mu_{t i}$ and $S_{i}=A_{i} \nabla u \cdot \nabla u$. The eddy viscosity for phase $i$ is $\mu_{t i}=\alpha_{2} \rho_{i} k / \omega$. The $k-\omega$ model parameters $\alpha_{1}, \alpha_{2}, \beta_{1}, \beta_{2}, \sigma_{1}$ and $\sigma_{2}$ are non-dimensional quantities. The symbol $\nabla$ denotes the gradient operator in the cross-section analyzed. Thus, in terms of the canonical base given by the Cartesian unit vectors $\mathbf{e}_{\mathrm{x}}$ and $\mathbf{e}_{\mathrm{y}}$, we have $\nabla=\frac{\partial}{\partial x} \mathbf{e}_{x}+\frac{\partial}{\partial y} \mathbf{e}_{y}$.

The model is completed by introducing boundary and interfacial conditions. The conditions on the symmetry boundary $\Gamma_{\mathrm{s}}$ are $\nabla u \cdot \mathbf{n}=0, \nabla k \cdot \mathbf{n}=0$ and $\nabla \omega \cdot \mathbf{n}=0$, where $\mathbf{n}$ is the outward pointing unit vector on $\Gamma_{\mathrm{s}}$. The pipe boundary $\Gamma_{\mathrm{c}}$ is split into $\Gamma_{\mathrm{cf}}$ and $\Gamma_{\mathrm{cg}}$, according to the phase which is in contact with the wall. Thus, the boundary conditions on $\Gamma_{\mathrm{c}}$ are $u=0, k=0$ and $\omega=\bar{\omega}_{\mathrm{c} i}$ on $\Gamma_{\mathrm{c} i}$, meaning that the prescribed value depends on the properties of the phase which is in contact with the pipe surface. It is well known that $\omega$ goes to infinity on smooth pipe walls. In order to avoid this singular behavior, we employ the same wall boundary condition implemented in the DEFT incompressible flow solver [29], which is given by

$\bar{\omega}_{\mathrm{c} i}=\frac{2 \mu_{i}}{\beta_{0} \rho_{i} Y_{\mathrm{p}}^{2}}$

where $\beta_{0}=0.072$ is a model constant and $Y_{\mathrm{p}}$ is the distance of the closest grid point to the wall.

At the interface $\Gamma_{\text {int }}$, we impose continuity of the shear stress and consider that the interface is smooth. Thus the turbulence kinetic energy at the interface is set as $k_{\text {int }}=0$. The value imposed for $\omega$ at the interface is denoted by $\bar{\omega}_{\text {int }}$ and is also computed using Eq. (4). However, Eq. (4) yields different values when computed from the different sides of the interface. Here we choose $\bar{\omega}_{\text {int }}$ to be the higher 
of these values in order to ensure that the interface is approximated as smooth when viewed from either side.

The problem described above can be recast in variational form as follows: find $u \in V_{u}, k \in V_{k}$ and $\omega \in V_{\omega}$, for any $\phi \in V_{\phi}$ and any $\varphi \in V_{\varphi}$, such that

$$
\begin{aligned}
F\left(\frac{\mathrm{d} p}{\mathrm{~d} z}, y_{\text {int }}\right) & =2 \int_{\Omega_{\mathrm{f}}} u \mathrm{~d} \Omega-Q_{\mathrm{f}} \\
G\left(\frac{\mathrm{d} p}{\mathrm{~d} z}, y_{\text {int }}\right) & =2 \int_{\Omega_{\mathrm{g}}} u \mathrm{~d} \Omega-Q_{\mathrm{g}}
\end{aligned}
$$

where,

$V_{u}=\left\{u \in H_{1}(\Omega), u=0\right.$ on $\left.\Gamma_{\mathrm{c}}\right\}$

$V_{k}=\left\{k \in H_{1}\left(\Omega_{\mathrm{f}} \cup \Omega_{\mathrm{g}}\right), k=0\right.$ on $\Gamma_{\mathrm{c}} k=0$ on $\left.\Gamma_{\text {int }}\right\}$

$V_{\omega}=\left\{\omega \in H_{1}\left(\Omega_{\mathrm{f}} \cup \Omega_{\mathrm{g}}\right), \omega=\bar{\omega}_{\mathrm{c} i}\right.$ on $\Gamma_{\mathrm{ci}} \omega=\bar{\omega}_{\text {int }}$ on $\left.\Gamma_{\text {int }}\right\}$

$V_{\phi}=\left\{\phi \in H_{1}(\Omega), \phi=0\right.$ on $\left.\Gamma_{\mathrm{c}}\right\}$

$V_{\varphi}=\left\{\varphi \in H_{1}\left(\Omega_{\mathrm{f}} \cup \Omega_{\mathrm{g}}\right), \varphi=0\right.$ on $\Gamma_{\mathrm{c}} \varphi=0$ on $\left.\Gamma_{\mathrm{int}}\right\}$

Note that the problem described above is not closed: the pressure gradient $\mathrm{d} p / \mathrm{d} z$ and the interface position $y_{\text {int }}$, which ultimately defines the domains $\Omega_{\mathrm{f}}$ and $\Omega_{\mathrm{g}}$, are unknown. The equations that close the model come from the requirement to meet the imposed flow rates $Q_{\mathrm{f}}$ and $Q_{\mathrm{g}}$, i.e.,

$Q_{\mathrm{f}}=2 \int_{\Omega_{\mathrm{f}}} u \mathrm{~d} \Omega$

$Q_{\mathrm{g}}=2 \int_{\Omega_{\mathrm{g}}} u \mathrm{~d} \Omega$

\section{Numerical techniques}

The solution is obtained by using an iterative process that combines two numerical techniques. The first is an external Newton-Raphson method aimed to adjust $y_{\text {int }}$ and $\mathrm{d} p / \mathrm{d} z$, in order to satisfy Eqs. (13) and (14). The second, which we call the flow solver, runs internally and involves the finite element solution of the non-linear problem given by Eqs. (5)-(7), for given values of $y_{\text {int }}$ and $\mathrm{d} p / \mathrm{d} z$.

\subsection{Newton-Raphson scheme}

Let us suppose that, for a given pair of $y_{\text {int }}$ and $\mathrm{d} p / \mathrm{d} z$, we have a numerical method to approximate and solve Eqs. (5)-(7), which will be described in the next section. Then we can compute the mismatch of the flow rates obtained for a given pair of $y_{\text {int }}$ and $\mathrm{d} p / \mathrm{d} z$ and the flow rates $Q_{\mathrm{f}}$ and $Q_{\mathrm{g}}$ imposed as problem data. The mismatch functions are
Introducing $x=\mathrm{d} p / \mathrm{d} z$ and $y=y_{\text {int }}$ to simplify notation, we can formulate the problem as a system of two non-linear equations whose solution $(x, y)$ must satisfy,

$$
\begin{aligned}
& F(x, y)=0 \\
& G(x, y)=0
\end{aligned}
$$

The Newton-Raphson method is used to compute the solution of the above non-linear system. If $x^{n}, y^{n}$ is the present approximate solution, the next approximation is computed according to

$$
\begin{aligned}
& x^{n+1}=x^{n}+\frac{\left(G \frac{\partial F}{\partial y}-F \frac{\partial G}{\partial y}\right)^{n}}{\left(\frac{\partial F}{\partial x} \frac{\partial G}{\partial y}-\frac{\partial G}{\partial x} \frac{\partial F}{\partial y}\right)} \\
& y^{n+1}=y^{n}+\frac{\left(F \frac{\partial G}{\partial x}-G \frac{\partial F}{\partial x}\right)^{n}}{\left(\frac{\partial F}{\partial x} \frac{\partial G}{\partial y}-\frac{\partial G}{\partial x} \frac{\partial F}{\partial y}\right)}
\end{aligned}
$$

Therefore, starting from an initial guess, the above equations provide an iterative algorithm to obtain a solution of the non-linear system given by Eqs. (17) and (18). The iterations proceed until the mismatch functions $F$ and $G$ are considered to be negligibly small. Before we proceed, note that we need to compute derivatives of $F$ and $G$ with respect to the unknowns $x$ and $y$. These derivatives are evaluated numerically, by using small increments $\Delta x$ and $\Delta y$. To compute them we have to evaluate $F$ and $G$ at $\left(x^{n}+\Delta x, y^{n}\right),\left(x^{n}, y^{n}+\Delta y\right)$ and $\left(x^{n}, y^{n}\right)$. This means that we need to solve Eqs. (5)-(7) three times for each Newton-Raphson iteration.

\subsection{Flow solver}

Given the interface position and the pressure gradient, the finite element method is used to obtain an approximate numerical solution of Eqs. (5)-(7).

A finite element mesh is constructed using a simple mesh generation routine based on the bipolar mapping described in detail in literature $[2-4,13]$. The mesh generator employed provides a selective refinement of mesh close to 
the pipe wall and at the gas-liquid interface. Here the bipolar mapping is used only to generate a suitable mesh. The problem is solved using the standard Cartesian co-ordinate system (not the bipolar co-ordinate system).

We use linear triangular finite elements to approximate the flow variables as $\hat{\mathrm{u}}=N_{\mathrm{p}} u_{\mathrm{p}}, \hat{k}=N_{\mathrm{p}} k_{\mathrm{p}}$ and $\hat{\omega}=N_{\mathrm{p}} \omega_{\mathrm{p}}$, where $N_{\mathrm{p}}$ are the finite element linear shape functions and $u_{\mathrm{p}}, k_{\mathrm{p}}$ and $\omega_{\mathrm{p}}$ are the corresponding nodal values. Because Eqs. (5)-(7) are non-linear, an iterative process is required. Thus, the velocity field is updated by solving the discretized counterpart of Eq. (5), i.e.,

$$
\begin{aligned}
& \sum_{i=f, g} \int_{\Omega_{i}} A_{i} \nabla N_{\mathrm{q}} \cdot \nabla \hat{u}^{n+1} \mathrm{~d} \Omega_{i} \\
& =-\sum_{i=f, g} \int_{\Omega_{i}} N_{\mathrm{q}} \frac{\mathrm{d} p}{\mathrm{~d} z} \mathrm{~d} \Omega_{i} \forall \text { free } \quad u_{\mathrm{q}}^{n+1}
\end{aligned}
$$

next, the kinetic energy is updated $\hat{k}^{n+1}$ by solving

$$
\begin{aligned}
& \sum_{i=f, g} \int_{\Omega_{i}} B_{i} \nabla N_{\mathrm{q}} \cdot \nabla \hat{k}^{n+1} \mathrm{~d} \Omega_{i} \\
& \quad+\sum_{i=f, g} \int_{\Omega_{i}} N_{\mathrm{q}} \beta_{2} \rho_{i} \hat{k}^{n+1} \hat{\omega}^{n} \mathrm{~d} \Omega_{i} \\
& =\sum_{i=f, g} \int_{\Omega_{i}} N_{\mathrm{q}} A_{i} \nabla \hat{u}^{n+1} \cdot \nabla \hat{u}^{n+1} \mathrm{~d} \Omega_{i} \quad \forall \text { free } \quad k_{\mathrm{q}}^{n+1}
\end{aligned}
$$

and finally, the new $\hat{\omega}$ is obtained by solving

$$
\begin{gathered}
\sum_{i=f, g} \int_{\Omega_{i}} C_{i} \nabla N_{\mathrm{q}} \cdot \nabla \hat{\omega}^{n+1} \mathrm{~d} \Omega_{i} \\
+\sum_{i=f, g} \int_{\Omega_{i}} N_{\mathrm{q}} \beta_{1} \rho_{i} \hat{\omega}^{n} \hat{\omega}^{n+1} \mathrm{~d} \Omega_{i} \\
=\sum_{i=f, g} \int_{\Omega_{i}} N_{\mathrm{q}} \alpha_{1} \frac{\hat{\omega}^{n}}{\hat{k}^{n+1}} A_{i} \nabla \hat{u}^{n+1} \\
\cdot \nabla \hat{u}^{n+1} \mathrm{~d} \Omega_{i} \quad \forall \text { free } \quad \omega_{\mathrm{q}}^{n+1}
\end{gathered}
$$

Eqs. (21)-(23) lead to symmetric systems of algebraic equations, which are solved by using a Jacobi-preconditioned conjugate gradient method. The process of solving Eqs. (21)-(23) is repeated until a prescribed convergence criterion is satisfied.

\section{Results and discussion}

In order to check the turbulence model employed and the computer implementation of the code, we have tested our procedures in a single phase problem, where we can compare our prediction for friction factor with corresponding theoretical and correlated experimental data. This

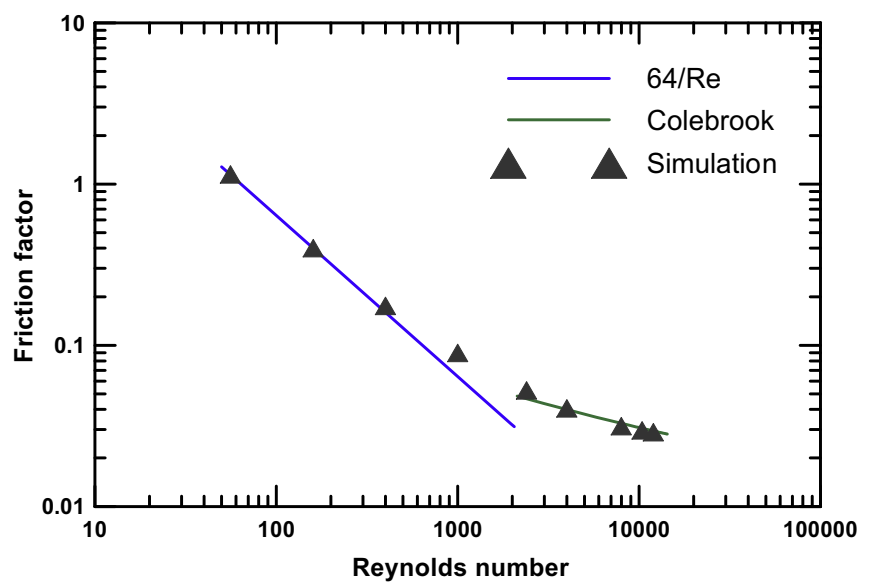

Fig. 2. Single-phase friction factor as a function of the Reynolds number: comparison of the present simulation with analytical solution and Colebrook's correlation.

Table 1

Liquid and gas superficial velocities of the test cases of two-phase laminarlaminar flow, with the hold-up and pressure gradient calculated by the CFD simulation

\begin{tabular}{lllll}
\hline Case & $U_{\mathrm{fs}}(\mathrm{cm} / \mathrm{s})$ & $U_{\mathrm{gs}}(\mathrm{cm} / \mathrm{s})$ & $\epsilon_{\mathrm{f}}$ & $\mathrm{d} p / \mathrm{d} z(\mathrm{~Pa} / \mathrm{m})$ \\
\hline 1 & 0.084 & 1.686 & 0.426 & -0.0140 \\
2 & 0.169 & 3.373 & 0.426 & -0.0280 \\
3 & 0.337 & 3.373 & 0.521 & -0.0422 \\
\hline
\end{tabular}

rather simple test consists of assigning the same fluid properties and flow rates for both phases. We have also relaxed the interface conditions on $k$ and $\omega$. This permits mimicking a single-phase computation using our two-phase computer code. As expected, the Newton-Raphson method found that the interface position is at the middle of the pipe, at $y_{\text {int }}=0$. The Newton-Raphson converged value for $\mathrm{d} p / \mathrm{d} z$ was used to compute the friction factor obtained in our numerical experiment. Fig. 2 presents a comparison of our data with the theoretical friction factor for laminar flow and with the friction factor predicted by Colebrook correlation for turbulent flow in a hydrodynamically smooth circular pipe. Note that our results compare well with the expected values for both laminar and turbulent flow, although the friction factor was over-estimated in the transition region.

A more general check for two-phase laminar-laminar flow has been carried out by comparison the numerical solution to the corresponding analytic solution of Biberg and Halvorsen [13], which presented the exact solution for the mean wall and interfacial shear stress, for given hold-up and pressure drop, in terms of simple algebraic

Table 2

Comparison of the interfacial and wall shear stresses predicted by the present simulation and the analytical solution given by Biberg and Halvorsen [13]

\begin{tabular}{lllllll}
\hline Case & $\bar{\tau}_{i}(\mathrm{mPa})$ Simulation & $\bar{\tau}_{i}(\mathrm{mPa}) \mathrm{B}-\mathrm{H}$ & $\bar{\tau}_{\mathrm{g}}(\mathrm{mPa})$ Simulation & $\bar{\tau}_{\mathrm{g}}(\mathrm{mPa}) \mathrm{B}-\mathrm{H}$ & $\bar{\tau}_{f}(\mathrm{mPa})$ Simulation & $\bar{\tau}_{f}(\mathrm{mPa}) \mathrm{B}-\mathrm{H}$ \\
\hline 1 & 0.1220 & 0.1220 & 0.1191 & 0.1193 & 0.2480 & 0.2481 \\
2 & 0.2434 & 0.2443 & 0.2360 & 0.2390 & 0.4957 & 0.4968 \\
3 & 0.3150 & 0.3180 & 0.3172 & 0.3216 & 0.7485 & 0.7495 \\
\hline
\end{tabular}


Table 3

Flow rates and pipe diameters $(d)$ of the experimental cases [32,33] compared with the simulation

\begin{tabular}{lllll}
\hline References & Case & $d(\mathrm{~cm})$ & $Q_{\mathrm{f}}\left(\mathrm{m}^{3} / \mathrm{h}\right)$ & $Q_{f}\left(\mathrm{~m}^{3} / \mathrm{h}\right)$ \\
\hline$[32]$ & A & 5.12 & 1.0 & 0.6 \\
& B & 5.12 & 2.0 & 0.6 \\
& C & 5.12 & 4.0 & 0.6 \\
& D & 5.12 & 6.0 & 0.6 \\
{$[33]$} & E & 2.10 & 0.3 & 0.024 \\
& F & 2.10 & 0.3 & 0.061 \\
& G & 2.10 & 0.3 & 0.090 \\
& H & 2.10 & 0.3 & 0.121 \\
\hline
\end{tabular}

expressions. For a given pair of liquid and gas superficial velocities, $U_{\mathrm{fs}}$ and $U_{\mathrm{gs}}$, the liquid hold-up $\epsilon_{\mathrm{f}}$ and the pres- sure gradient $\mathrm{d} p / \mathrm{d} z$ are calculated by our CFD simulation, for two-phase flow in a $5.12 \mathrm{~cm}$ internal diameter pipe at standard conditions. The calculated hold-up and pressure gradient are three test cases given in Table 1. The liquid wetted angle $\delta_{\mathrm{f}}$ is related to the liquid hold-up through the following expression:

$\epsilon_{\mathrm{f}}=\frac{1}{\pi}\left(\delta_{\mathrm{f}}-\frac{1}{2} \sin 2 \delta_{\mathrm{f}}\right)$

For two-phase laminar-laminar flow in a horizonal circular pipe, Biberg and Halvorsen [13] obtained the following remarkably simple and exact expression for the mean interfacial shear stress:
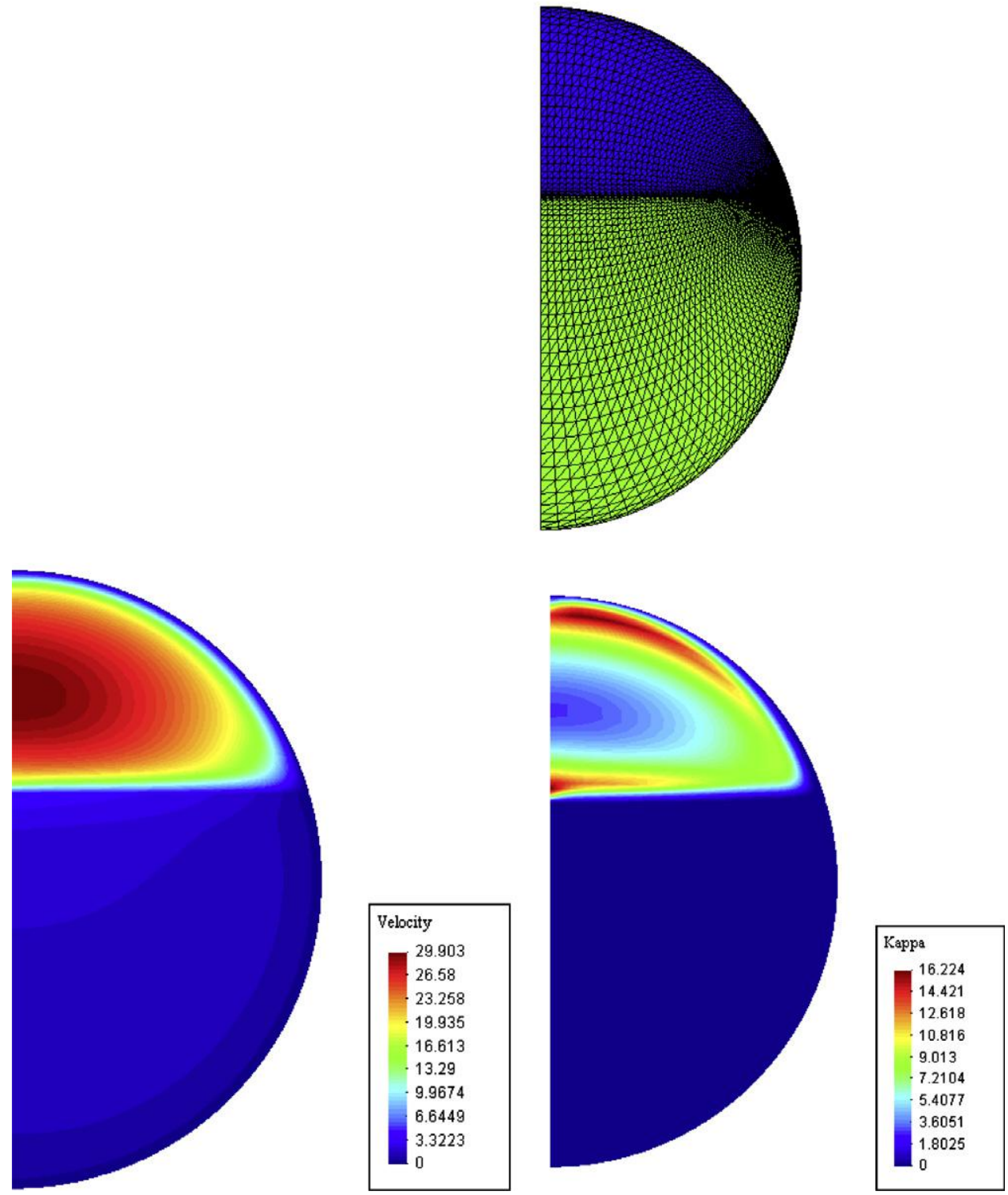

Fig. 3. Mesh, velocity and turbulent kinetic energy for case C, $Q_{\mathrm{f}}=0.6 \mathrm{~m}^{3} / \mathrm{h}$ and $Q_{\mathrm{g}}=4.0 \mathrm{~m}^{3} / \mathrm{h}$. 
$\bar{\tau}_{i}=\frac{R}{2}\left(\frac{\mathrm{d} p}{\mathrm{~d} z}\right) \frac{\left[\mu_{\mathrm{g}}\left(\sin \delta_{\mathrm{f}}-\delta_{\mathrm{f}} \cos \delta_{\mathrm{f}}\right)-\mu_{\mathrm{f}}\left(\sin \delta_{\mathrm{g}}-\delta_{\mathrm{g}} \cos \delta_{\mathrm{g}}\right)\right]}{\mu_{\mathrm{g}} \delta_{\mathrm{f}}+\mu_{\mathrm{f}} \delta_{\mathrm{g}}}$

Biberg and Halvorsen [13] also obtained the following simple expressions for the mean wall interfacial stresses:

$\bar{\tau}_{\mathrm{g}}=\frac{R}{2}\left(-\frac{\mathrm{d} p}{\mathrm{~d} z}\right)\left(\frac{\delta_{\mathrm{g}}-(1 / 2) \sin 2 \delta_{\mathrm{g}}}{\delta_{\mathrm{g}}}\right)-\bar{\tau}_{i} \frac{\sin \delta_{\mathrm{g}}}{\delta_{\mathrm{g}}}$

$\bar{\tau}_{\mathrm{f}}=\frac{R}{2}\left(-\frac{\mathrm{d} p}{\mathrm{~d} z}\right)\left(\frac{\delta_{\mathrm{f}}-(1 / 2) \sin 2 \delta_{\mathrm{f}}}{\delta_{\mathrm{f}}}\right)+\bar{\tau}_{i} \frac{\sin \delta_{\mathrm{f}}}{\delta_{\mathrm{f}}}$

The mean interfacial and wall shear stresses computed by the present CFD simulation are compared with the values obtained by using the exact algebraic expressions from Bi-
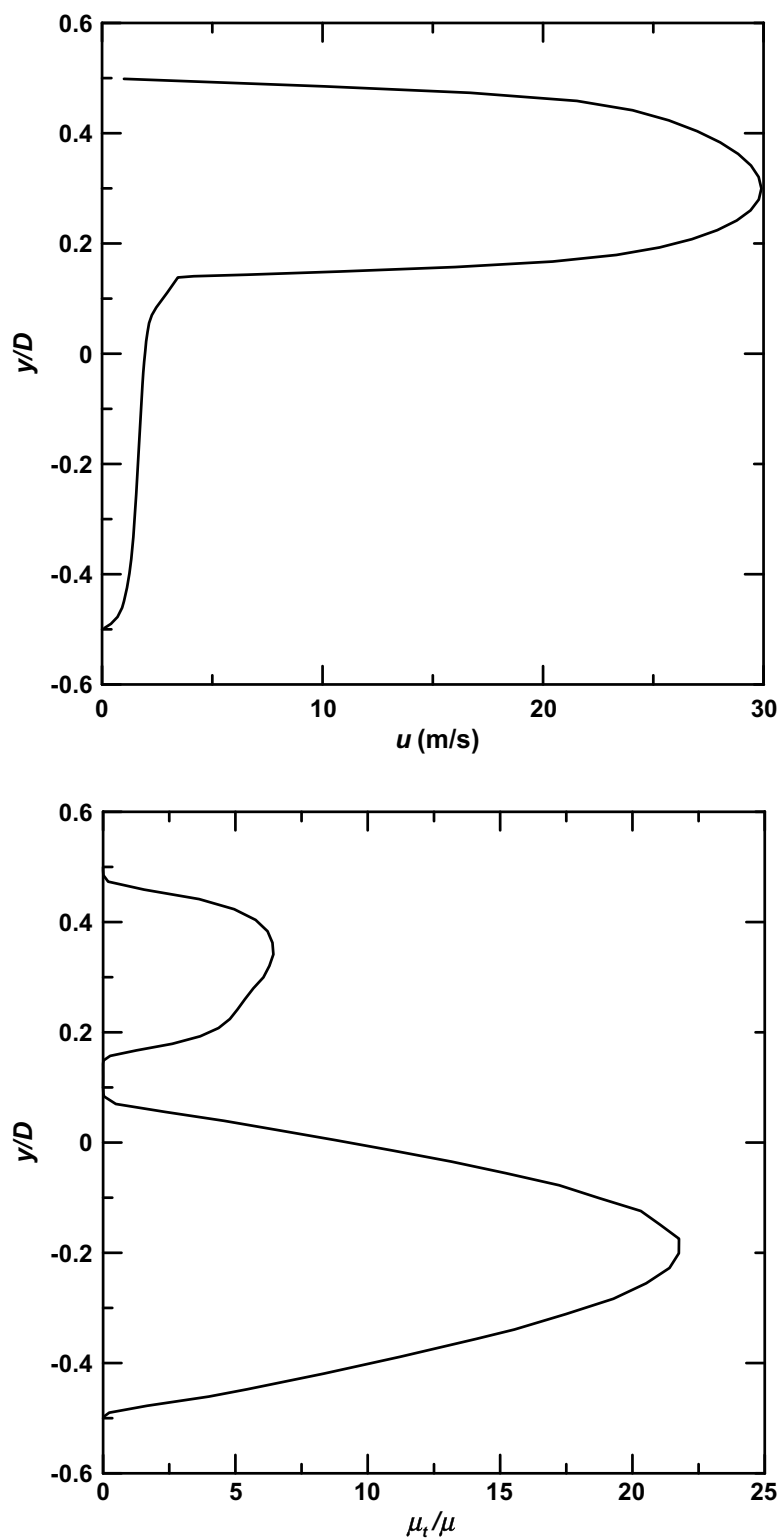

berg and Halvorsen [13], Eqs. (25)-(27), for the conditions given in Table 1. It can be seen from Table 2 that the agreement between the numerical and analytic solutions is excellent.

In what follows we present results for the interface position at various flow conditions, comparing the numerical and our own experimental data obtained at the Nuclear Engineering Institute (IEN/CNEN), Brazil [30-32], and the experimental data by Masala [33]. The experiments in IEN were conducted in the two-phase test rig consisting of a venturi mixer, a horizontal tube, an expansion reservoir and an air water separation tank. The horizontal tube is a $5 \mathrm{~m}$ long stainless steel 316 with an inner diameter of $0.0512 \mathrm{~m}$, followed by a short tube $0.6 \mathrm{~m}$ long transparent extruded acrylic with the same inner diameter. The gas-
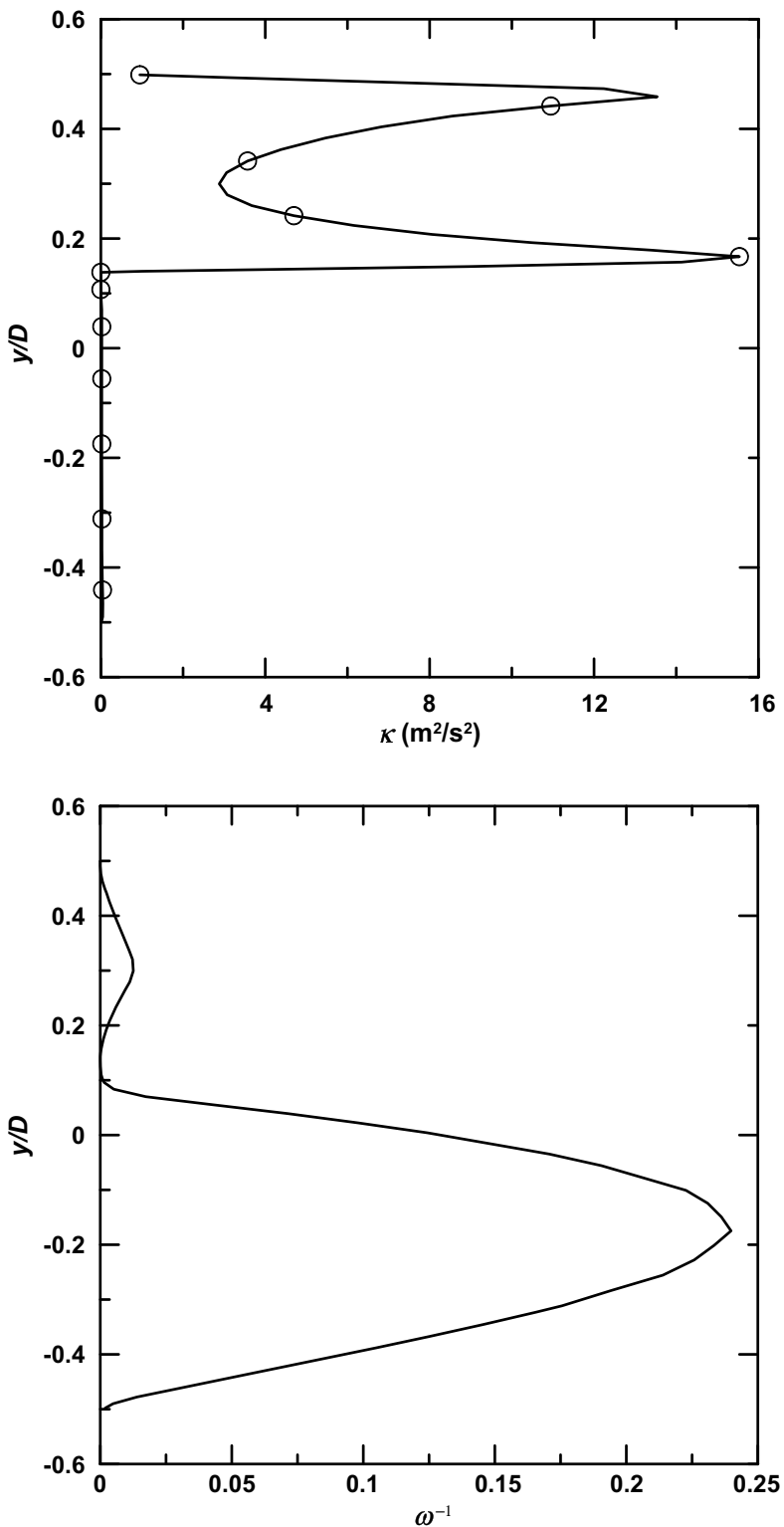

Fig. 4. Velocity, eddy viscosity, turbulent kinetic energy, inverse of the specific dissipation rate distribution at the vertical symmetry line for case C, $Q_{\mathrm{f}}=0.6 \mathrm{~m}^{3} / \mathrm{h}$ and $Q_{\mathrm{g}}=4.0 \mathrm{~m}^{3} / \mathrm{h}$. 

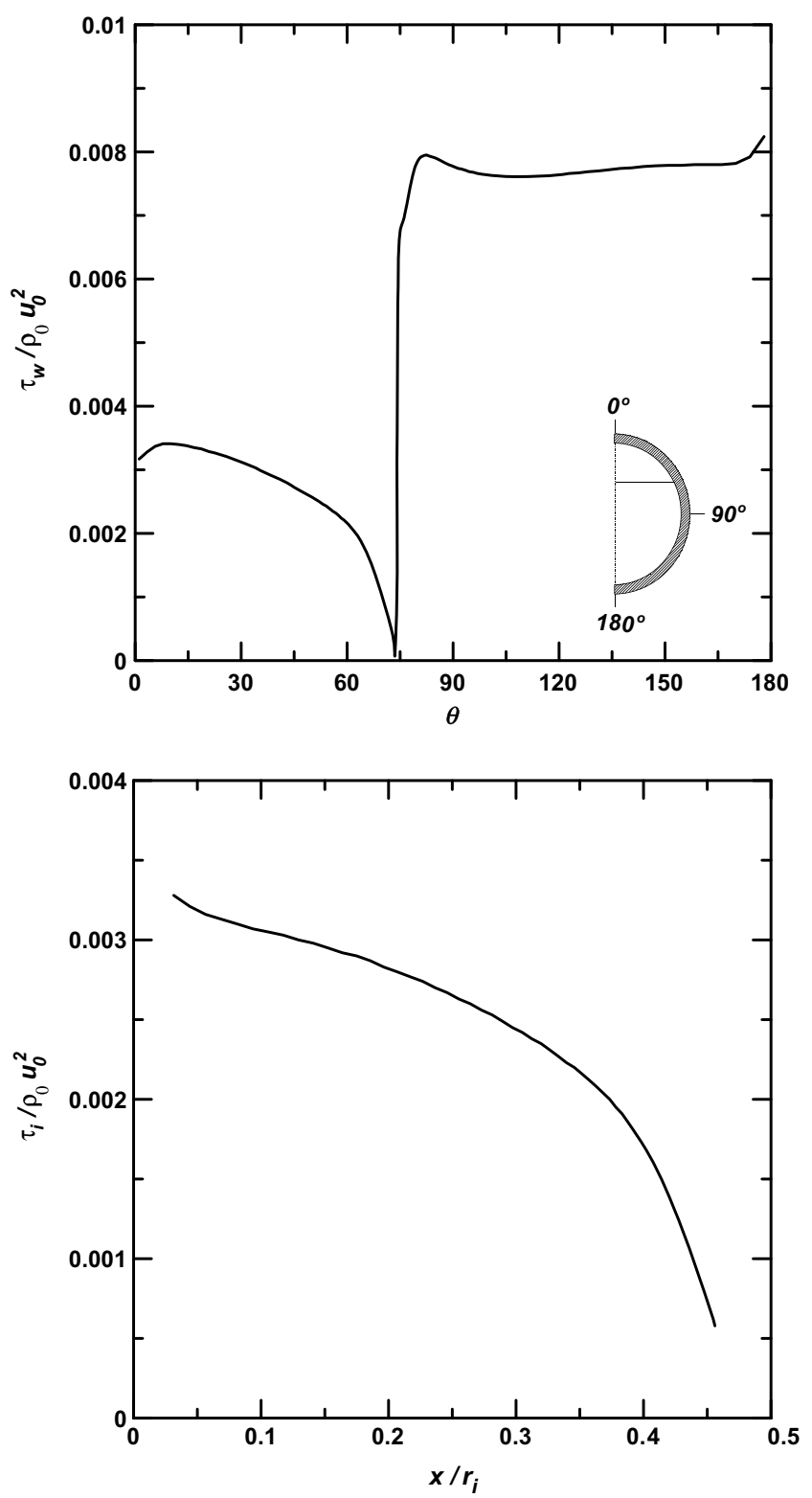

Fig. 5. Wall and interfacial shear stress distributions for case $\mathrm{C}$, $Q_{\mathrm{f}}=0.6 \mathrm{~m}^{3} / \mathrm{h}$ and $Q_{\mathrm{g}}=4.0 \mathrm{~m}^{3} / \mathrm{h}$.
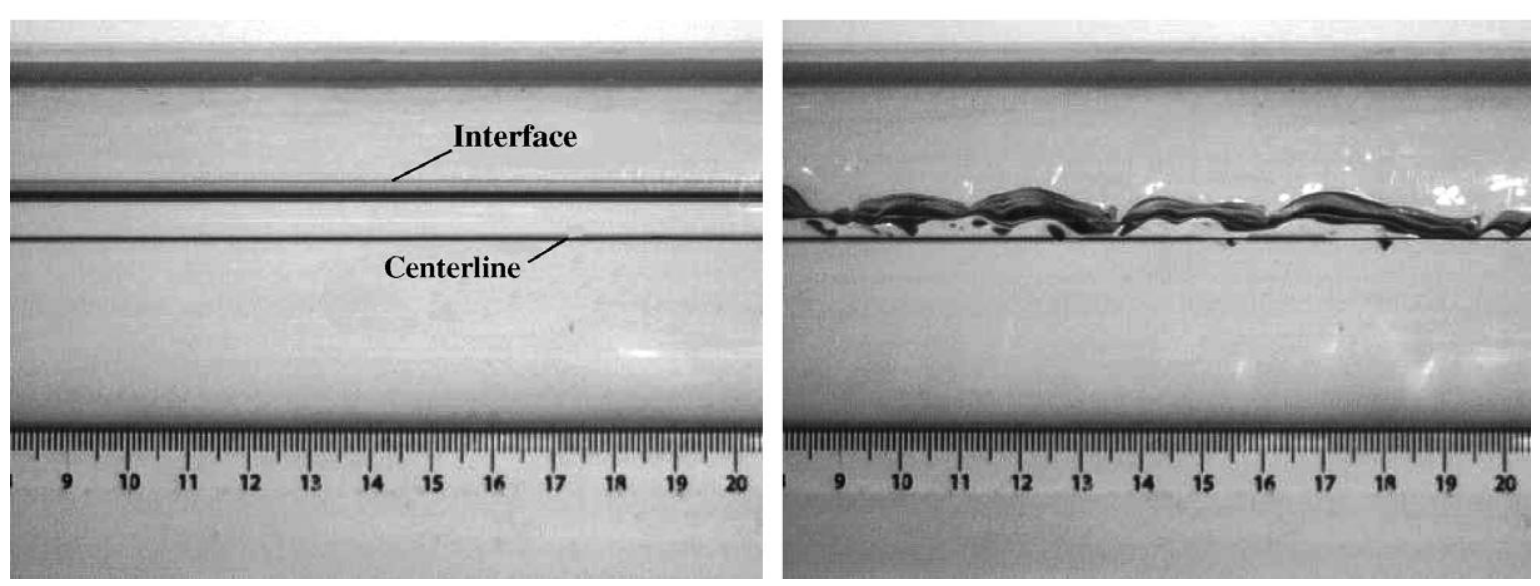

Fig. 6. Flow visualization of stratified flow in the horizontal tube: (a) $Q_{\mathrm{f}}=0.6 \mathrm{~m}^{3} / \mathrm{h}$ and $Q_{\mathrm{g}}=4.0 \mathrm{~m}^{3} / \mathrm{h}$; (b) $Q_{\mathrm{f}}=0.6 \mathrm{~m}^{3} / \mathrm{h}$ and $Q_{\mathrm{g}}=6.0 \mathrm{~m}^{3} / \mathrm{h}$. liquid interface position was measured by using the pulseecho ultrasonic technique. The ultrasound pulse discharged from an emitter-receiver transducer, placed at bottom of the tube, is transmitted through the water and then reflected back to the same transducer from the air-water or tube wall-water interfaces. The transit time between the ultrasonic pulse and echo signals provides an accurate measurement of the liquid film thickness. A detailed description of the test section can be found in Faccini et al. [30]. We have also compared our data with results obtained using the model of Taitel and Dukler [1]. The flow rates and pipe diameters used in the experiments and numerical computations are presented in Table 3 . In all simulations, the fluid properties at $1 \mathrm{~atm}$ and $25^{\circ} \mathrm{C}$ are used: $\mu_{\mathrm{f}}=8.6 \times 10^{-4} \mathrm{~Pa} \mathrm{~s} ; \quad \mu_{\mathrm{g}}=1.85 \times 10^{-5} \mathrm{~Pa} \mathrm{~s} ; \quad \rho_{\mathrm{f}}=$ $996 \mathrm{~kg} / \mathrm{m}^{3} ; \rho_{\mathrm{g}}=1.18 \mathrm{~kg} / \mathrm{m}^{3}$.

We have performed numerical simulations for all the cases presented in Table 3. Fig. 3 shows a typical result of mesh, velocity and kinetic energy. Fig. 4 shows example plots of velocity, eddy viscosity, $k$ and $1 / \omega$ distributions on the vertical symmetry line of the pipe for the case with $Q_{\mathrm{f}}=0.6 \mathrm{~m}^{3} / \mathrm{h}$ and $Q_{\mathrm{g}}=4 \mathrm{~m}^{3} / \mathrm{h}$. Fig. 5 shows typical results of wall and interfacial shear stress for the same case. Fig. 6 shows typical pictures taken with a high-speed camera during the IEN experiments [32]. Despite the fact that the interfaces shown in Fig. 6 are located in the upper part of the horizontal tube, it can be observed that the flow regime is stratified for the flow rates considered. Note that the model idealization depicted in Fig. 1 has a better match with the flow pattern presented in Fig. 6a than with that shown in Fig. 6b. A wavy interface such as that observed in Fig. 6b may require non-smooth interfacial conditions in order to obtain better results from the numerical model. This will be investigated in a future work. Fig. 7 shows the nondimensionalized results for the interface position $y_{\text {int }} / d$ as a function of volumetric quality $\beta=Q_{\mathrm{g}} /\left(Q_{\mathrm{f}}+Q_{\mathrm{g}}\right)$, comparing the present simulation with the McMaster experiments performed by Masala [33] and with numerical predictions obtained using the model of Taitel and Dukler 


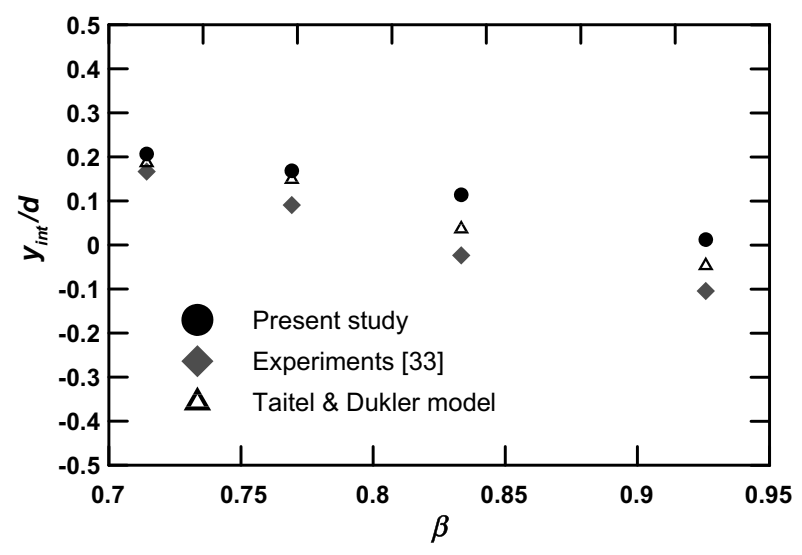

Fig. 7. Dimensionless interface position as a function of the volumetric quality: comparison of present simulation with experimental data of Masala [33] and numerical results from the Taitel and Dukler [1] model.

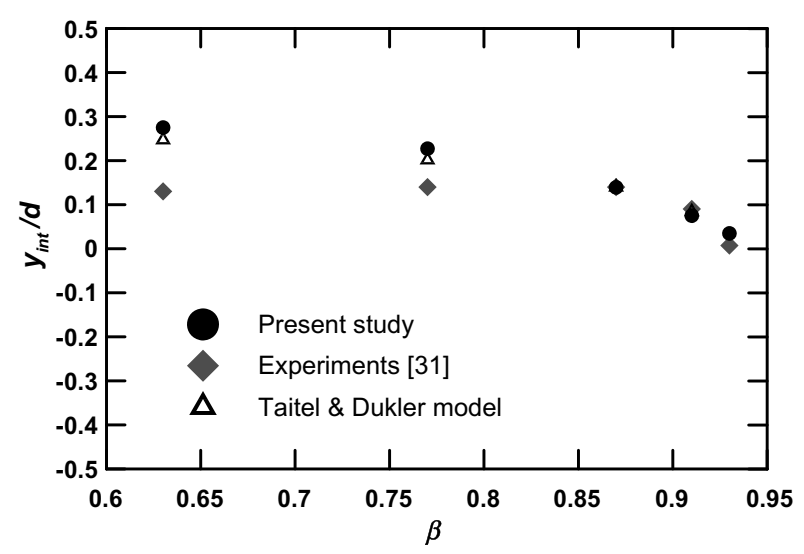

Fig. 8. Dimensionless interface position as a function of the volumetric quality: comparison of present simulation with experiments performed at IEN [32] and numerical results from the Taitel and Dukler [1] model.

[1]. Fig. 8 shows similar results, this time comparing the present simulation with the experiments performed at IEN [31]. Figs. 7 and 8 show that the results of the present simulation are in reasonable agreement with both sets of experimental data and the predictions of the Taitel and Dukler model. It should be noted that the Taitel-Dukler model treats the gas as if it was in single-phase flow over a frozen liquid with a smooth surface, which is also assumed in our CFD model. The influence of the interfacial boundary conditions, especially that of $k$ and $\omega$, on the solutions should be further investigated. It is interesting to note also that Masala's data [33], for volumetric qualities higher than 0.80 , have at least one of the phases undergoing transition. As we have noticed in the singlephase simulation test, the numerical model over-estimates the friction factor at transition. This may explain some discrepancy observed between the present simulation results and Masala's experimental data on that volumetric quality range.

\section{Conclusions}

In this paper we proposed physical and numerical models for stratified two-phase flows in horizontal pipes, comparing results with available experimental data. We have also compared our simulation data with those obtained using the model of Taitel and Dukler [1]. The results indicate that the $k-\omega$ model is suitable for the numerical simulation of such flows. However, a better understanding on how to impose interfacial values for $k$ and $\omega$ is needed before we can expect to obtain better agreement with experimental data of stratified wavy two-phase flow. The computational code is being parallelized to run on a Beowulf type cluster. It is expected that with a parallel version of the code we will be able to re-calculate the cases presented here in much finer meshes, with possible improvement of the quality of the numerical results.

\section{Acknowledgements}

J.L.H. Faccini is grateful to IAEA for the donation of the instrumentation, and to Silvia B.G. Cesar for technical assistance. P.A.B de Sampaio acknowledges the support of CNPq Grant 301045/2003-8. J. Su acknowledges the support of CNPq and FAPERJ. The authors are grateful to Professor Jen-Shih Chang for kindly providing the ultrasonic pulser/receiver and helpful discussions on ultrasonic pulse-echo techniques. The authors would like to thank the reviewer for valuable comments and suggestions.

\section{References}

[1] Y. Taitel, A.E. Dukler, Model for predicting flow regime transitions in horizontal and near horizontal gas-liquid flow, AIChE J. 22 (1976) 591-595.

[2] O. Shoham, Y. Taitel, Stratified turbulent-turbulent gas-liquid flow in horizontal and inclined pipes, AIChE J. 30 (1984) 377-385.

[3] R. Issa, Prediction of turbulent, stratified, two-phase flow in inclined pipes and channel, Int. J. Multiphase Flow 14 (1988) 141-154.

[4] C.H. Newton, M. Behnia, Numerical calculation of turbulent stratified gas-liquid pipe flows, Int. J. Multiphase Flow 26 (2000) 327-337.

[5] C.H. Newton, M. Behnia, Estimation of wall shear stress in horizontal gas-liquid stratified flow, AIChE J. 42 (1996) 2369-2373.

[6] F. Meknassi, R. Benkirane, A. Liné, L. Masbernat, Numerical modeling of wavy stratified two-phase flow in pipes, Chem. Eng. Sci. 55 (2000) 4681-4697.

[7] C.H. Newton, M. Behnia, A numerical model of stratified wavy gasliquid pipe flow, Chem. Eng. Sci. 56 (2001) 6851-6861.

[8] P.A. Berthelsen, T. Ytrehus, Calculation of stratified wavy two-phase flow in pipes, Int. J. Multiphase Flow 31 (2005) 571-592.

[9] S. Ghorai, K.D.P. Nigam, CFD modeling of flow profiles and interfacial phenomena in two-phase flow in pipes, Chem. Eng. Process. 45 (2006) 55-65.

[10] A.A. Mouza, S.V. Paras, A.J. Karabelas, Cfd code application to wavy stratified gas-liquid flow, Chem. Eng. Res. Design 79 (A5) (2001) 561-568.

[11] M. Akai, A. Inoue, S. Aoki, K. Endo, A co-current stratified airmercury flow with wavy interface, Int. J. Multiphase Flow 6 (3) (1980) 173-190. 
[12] M. Akai, A. Inoue, S. Aoki, The prediction of stratified 2-phase flow with a 2-equation model of turbulence, Int. J. Multiphase Flow 7 (1) (1981) 21-39.

[13] D. Biberg, G. Halvorsen, Wall and interfacial shear stress in pressure driven two-phase laminar stratified pipe flow, Int. J. Multiphase Flow 26 (10) (2000) 1645-1673.

[14] R. Banerjee, K.M. Isaac, A study to determine vapor generation from the surface of gasoline flowing in an inclined channel using a continuous thermodynamics approach, Num. Heat Transfer. Part A. Appl. 50 (8) (2006) 705-729.

[15] Y.F. Yap, J.C. Chai, K.C. Toh, T.N. Wong, Modeling the flows of two immiscible fluids in a three-dimensional square channel using the levelset method, Num. Heat Transfer. Part A. Appl. 49 (9) (2006) 893-904.

[16] L.L. Zheng, H. Zhang, An adaptive level set method for movingboundary problems: application to droplet spreading and solidification, Num. Heat Transfer. Part B. Fundamentals 37 (4) (2000) 437-454

[17] G. Son, Efficient implementation of a coupled level-set and volume-offluid method for three-dimensional incompressible two-phase flows, Num. Heat Transfer. Part B. Fundamentals 43 (6) (2003) 549-565.

[18] H. Ki, P.S. Mohanty, J. Mazumder, A numerical method for multiphase incompressible thermal flows with solid-liquid and liquid-vapor phase transformations, Num. Heat Transfer. Part B. Fundamentals 48 (2) (2005) 125-145.

[19] C.F. Tai, W. Shyy, Multigrid computations and conservation law treatment of a sharp interface method, Num. Heat Transfer. Part B. Fundamentals 48 (5) (2005) 405-424.

[20] K. Ghosh, A. Mukhopadhyay, S. Sen, D. Sanyal, A sphericosymmetric VOF approach for investigating immiscible two-phase systems with one liquid phase, Num. Heat Transfer. Part A. Appl. 50 (10) (2006) 949-974.

[21] J. Rice, A. Faghri, New computational method for free surface problems, Num. Heat Transfer. Part B. Fundamentals 49 (5) (2006) 409-436.

[22] Y.F. Yap, J.C. Chai, T.N. Wong, K.C. Toh, H.Y. Zhang, A global mass correction scheme for the level-set method, Num. Heat Transfer. Part B. Fundamentals 50 (5) (2006) 455-472.
[23] J.F. Wu, V.K. Dhir, J.L. Qian, Numerical simulation of subcooled nucleate boiling by coupling level-set method with moving-mesh method, Num. Heat Transfer. Part B. Fundamentals 51 (6) (2007) $535-563$.

[24] M. Fulgosi, D. Lakehal, S. Banerjee, V. De Angelis, Direct numerical simulation of turbulence in a sheared air-water flow with a deformable interface, J. Fluid Mech. 482 (2003) 319-345.

[25] S. Reboux, P. Sagaut, D. Lakehal, Large-eddy simulation of sheared interfacial flow, Phys. Fluids 18 (10) (2006).

[26] D. Wilcox, Turbulence Modelling for CFD, DCW Industries, La Cañada, CA, 2000.

[27] F.R. Menter, M. Kunz, R. Langtry, Ten years of industrial experience with the SST turbulence model, in: K. Hanjalic, Y. Nagano, M. Tummers (Eds.), Turbulence, Heat and Mass Transfer, vol. 4, Begell House, 2003.

[28] F. Menter, Influence of freestream values of on $k-\omega$ turbulence model predictions, AIAA J. 30 (1992) 1657-1659.

[29] G. Segal, M. Zijlema, R. van Nooyen, C. Moulinec, User Manual of the DEFT Incompressible Flow Solver, Delft University of Technology, 2006.

[30] J.L.H. Faccini, J. Su, G.D. Harvel, J.S. Chang, An advanced ultrasonic technique for flow and void fraction measurements of two-phase flow, in: Proceedings of the 12th International Conference on Nuclear Engineering (ICONE12), Arlington, Virginia, Washington, DC, 2004.

[31] J.L.H. Faccini, J. Su, Measurement of film thickness and void fraction in stratified horizontal gas-liquid flow using an advanced ultrasonic technique, in: Proceedings of the 2005 International Nuclear Atlantic Conference (INAC2005), Santos, Brazil, 2005.

[32] J.L.H. Faccini, P.A.B. de Sampaio, J. Su, Assessment of intermittent two-phase flow using a high-speed visualization technique, in: Proceedings of 2007 International Nuclear Atlantic Conference (INAC2007), Santos, Brazil, 2007.

[33] T. Masala, High-speed ultrasonic pulse-echo system for two-phase flow measurements, Master's Thesis, McMaster University, 2004. 